\title{
Patient-Reported Comorbidity and Survival in Older Adults with Cancer
}

\author{
Grant R. Williams (D), a Aluson M. Deal, ${ }^{b}$ Jennifer L. Lund, ${ }^{b}$ YunKyung Chang, ${ }^{b}$ Hyman B. Muss, ${ }^{b}$ Mackenzi Pergolotti, \\ Emily J. Guerard, ${ }^{\text {d }}$ Shlomit Strulov Shachar, ${ }^{\text {b,e }}$ Yue Wang, ${ }^{\text {b }}$ Kelly Kenzik, ${ }^{a}$ Hanna K. Sanoff (D ${ }^{b}$ \\ anstitute for Cancer Outcomes \& Survivorship, University of Alabama at Birmingham, Birmingham, Alabama, USA; ${ }^{b}$ UNC Lineberger \\ Comprehensive Cancer Center, Chapel Hill, North Carolina, USA; 'Department of Occupational Therapy, Colorado State University, Fort \\ Collins, Colorado, USA; ${ }^{\mathrm{d} D i v i s i o n}$ of Hematology and Oncology, University of Wisconsin, Madison, Wisconsin, USA; ${ }^{\mathrm{e} D i v i s i o n}$ of Oncology, \\ Rambam Health Care Campus, Haifa, Israel \\ Disclosures of potential conflicts of interest may be found at the end of this article.
}

Key Words. Comorbidity • Multimorbidity • Cancer • Aging • Geriatric oncology

\section{ABSTRACT}

Background. Our ability to optimize the care of older adults with cancer and comorbid illnesses is insufficient because most clinical trials lack systematic measurement. The primary purpose of this study was to evaluate the association between patient-reported comorbidity and all-cause mortality using various comorbidity scoring algorithms.

Materials and Methods. The Carolina Senior Registry was linked with the North Carolina Central Cancer Registry to obtain mortality data. Comorbidity was assessed using the patientreported Older Americans Resources and Services Questionnaire subscale that assesses 13 specific conditions and the degree to which each impairs activities. Multivariable Cox proportional hazard regression models were used to evaluate the association between comorbidities and all-cause mortality.

Results. The study sample included 539 patients; the median age was 72 years, $72 \%$ were female, and $47 \%$ had breast cancer.
Overall, 92\% reported $\geq 1$ comorbid condition, with a mean of 2.7 conditions (range $0-10$ ), with arthritis and hypertension the most common (52\% and 50\%, respectively). Approximately $60 \%$ reported a functional limitation related to comorbidity. After adjusting for time from diagnosis to geriatric assessment, age, cancer type, and stage, the risk of death increased by $5 \%$ for each unit increase in comorbidity burden score (adjusted hazard ratio $[\mathrm{HR}]=1.05,95 \%$ confidence interval $[\mathrm{Cl}]$ : $1.01-$ 1.10) and $12 \%$ for each comorbid condition impacting function ( $\mathrm{HR}=1.12,95 \% \mathrm{Cl}: 1.02-1.23)$.

Conclusion. Comorbid conditions in older adults with cancer are highly prevalent and associated with all-cause mortality, particularly those conditions that impair function. Routine comorbidity assessment should be included in clinical trials and can be measured via a simple one-page patient-reported questionnaire. The Oncologist 2018;23:433-439

Implications for Practice: In order to optimize and personalize the care of older adults with cancer, systematic measurement of comorbidities is necessary in both clinical trials and routine practice. Patient-reported comorbid conditions in older adults with cancer are highly prevalent and are associated with increased risk of all-cause mortality, particularly for those conditions that impair function. Comorbidity can be systematically measured via a one-page patient-reported questionnaire and should be incorporated into future clinical trials and considered for use in oncology clinics to aid in assessing older adults with cancer.

\section{INTRODUCTION}

Cancer disproportionately impacts older adults, and given changing demographics, the number of older adults with cancer is increasing dramatically [1]. As our population ages, more individuals are affected by chronic conditions, and the importance of comorbid conditions in older adults with cancer is an area of growing importance [2]. Comorbidity, defined as a medical condition that exists along with an index condition, is highly prevalent, with as many as two thirds of Medicare beneficiaries with two or more medical conditions and nearly $25 \%$ with four or more chronic conditions [3]. Older adults with cancer have an increased burden of comorbidity compared with noncancer populations [4]. Comorbid conditions in oncology frequently impact cancer treatment decisions and are associated with allcause mortality and increased treatment-related toxicity and adverse events $[5,6]$.

Although the importance of comorbidity has been repeatedly demonstrated within oncology, comorbid conditions are rarely measured or routinely reported in clinical trials [6]. 
Evidence-based approaches to treating multimorbid patients with cancer are severely limited due to the frequent exclusion of patients with comorbid illnesses and lack of systematic assessments of comorbid conditions in clinical trials. Furthermore, no systematic method of comorbidity assessment is used in routine clinical practice, besides for what is typically collected within the routine history and physical. Validated tools provide a more systematic description of the severity and burden of comorbid illnesses, yet many of the existing comorbidity indices were developed for research purposes and are not designed for use in clinical practice.

Patient-reported outcomes are increasingly being used to assess quality of life, symptoms, and functional status in cancer clinical trials and provide a potential avenue for assessing comorbid conditions without additional burden to physicians or office staff [7]. The Older Americans Resources and Services (OARS) program developed several valid and reliable tools for use in older adults, including the patient-reported OARS comorbidity questionnaire [8]. The OARS questionnaire assesses the presence of 13 common comorbidities and additionally inquires about the degree to which the individual comorbid conditions interfere with activities. This brief tool has been incorporated into several geriatric assessments for use in older adults with cancer, yet the prevalence, scoring, and impact of patient-reported comorbidity on mortality using the OARS subscale remains poorly understood.

The primary purpose of this study was to evaluate the prevalence and potential impact of patient-reported comorbidity on all-cause mortality using the OARS comorbidity scale. In particular, we evaluated associations between different scoring algorithms of the OARS comorbidity scale and all-cause mortality to provide some guidance on how to operationalize patientreported comorbidity.

\section{MATERIALS AND METHODS}

\section{Patient Population}

We utilized a unique linkage of the Carolina Senior Registry (CSR), an institutional registry (NCT01137825) that contains geriatric assessment (GA) data, with the North Carolina Central Cancer Registry (NCCCR) to obtain mortality data. The CSR was developed in 2009 as a registry to collect GA information on older adults $(65+)$ with a diagnosis of cancer [9]. The CSR uses a brief, cancer-specific GA that was developed by Hurria et al. to better characterize the "functional age" of older patients with cancer [10]. The present study was limited to the 546 patients within the CSR who were recruited at the North Carolina Cancer Hospital between 2009 and 2014 and were linked to the NCCCR [11]. Survival status was determined through linking to the National Death Index, Social Security Death Index, and North Carolina State Center for Vital Statistics, and was available through August 2015. The NCCCR collects data on all cancers diagnosed in the state of North Carolina including date of diagnosis, cancer type, and stage according to the American Joint Committee on Cancer 6th Edition. This study was approved by The University of North Carolina Institutional Review Board.

\section{Comorbidity Measurement}

Comorbidity was assessed using a patient-reported version of the OARS Physical Health subscale [8]. The OARS comorbidity
Table 1. Patient characteristics $(n=539)$

\begin{tabular}{|c|c|}
\hline Characteristics & $n(\%)$ \\
\hline \multicolumn{2}{|l|}{ Age, years } \\
\hline Median (range) & $72(65-100)$ \\
\hline $65-69$ & $206(38)$ \\
\hline $70-74$ & $154(29)$ \\
\hline $75-79$ & $90(17)$ \\
\hline $80+$ & $89(17)$ \\
\hline \multicolumn{2}{|l|}{ Gender } \\
\hline Female & $388(72)$ \\
\hline Male & $151(28)$ \\
\hline \multicolumn{2}{|l|}{ Race } \\
\hline White & $457(85)$ \\
\hline Other & $82(15)$ \\
\hline \multicolumn{2}{|l|}{ Cancer type } \\
\hline Breast & $253(47)$ \\
\hline Lung and bronchus & $65(12)$ \\
\hline Hematologic malignancy & $65(12)$ \\
\hline Gastrointestinal & $40(7)$ \\
\hline Genitourinary & $40(7)$ \\
\hline Head and neck & $31(6)$ \\
\hline Other & $45(8)$ \\
\hline \multicolumn{2}{|l|}{ Cancer stage } \\
\hline Stage $0 / 1$ & $161(35)$ \\
\hline Stage II & $129(25)$ \\
\hline Stage III & $85(17)$ \\
\hline Stage IV & 95 (19) \\
\hline Unstaged/unknown & $41(8)$ \\
\hline \multicolumn{2}{|l|}{ Time from diagnosis to complete GA } \\
\hline Within 90 days & $176(33)$ \\
\hline 91 days to 1 year & $118(22)$ \\
\hline 1 year to 3 years & $107(20)$ \\
\hline$>3$ years & $138(26)$ \\
\hline \multicolumn{2}{|l|}{ Educational level } \\
\hline HS graduate or less & $264(49)$ \\
\hline Associate/bachelor's & $147(27)$ \\
\hline Advanced degree & $125(23)$ \\
\hline $\begin{array}{l}\text { Total no. of comorbid conditions } \\
\text { Median (range) }\end{array}$ & $2(0-10)$ \\
\hline $\begin{array}{l}\text { No. of comorbid conditions } \\
\text { interfering with activities } \\
\text { Median (range) }\end{array}$ & $1(0-7)$ \\
\hline $\begin{array}{l}\text { Comorbidity burden score } \\
\text { Median (range) }\end{array}$ & $3(0-17)$ \\
\hline
\end{tabular}

Abbreviations: GA, geriatric assessment; HS, high school.

scale includes information regarding 13 specific comorbid conditions and the degree to which each impairs the participant's activities (not at all, somewhat, or a great deal) [8]. Individual comorbid conditions assessed include other cancers or leukemia, arthritis or rheumatism, glaucoma, emphysema or chronic bronchitis, high blood pressure, heart disease, circulation trouble in arms or legs, diabetes, stomach or intestinal disorders, osteoporosis, chronic liver or kidney disease, stroke, or depression. 


\section{A Prevalence of Comorbid Conditions}

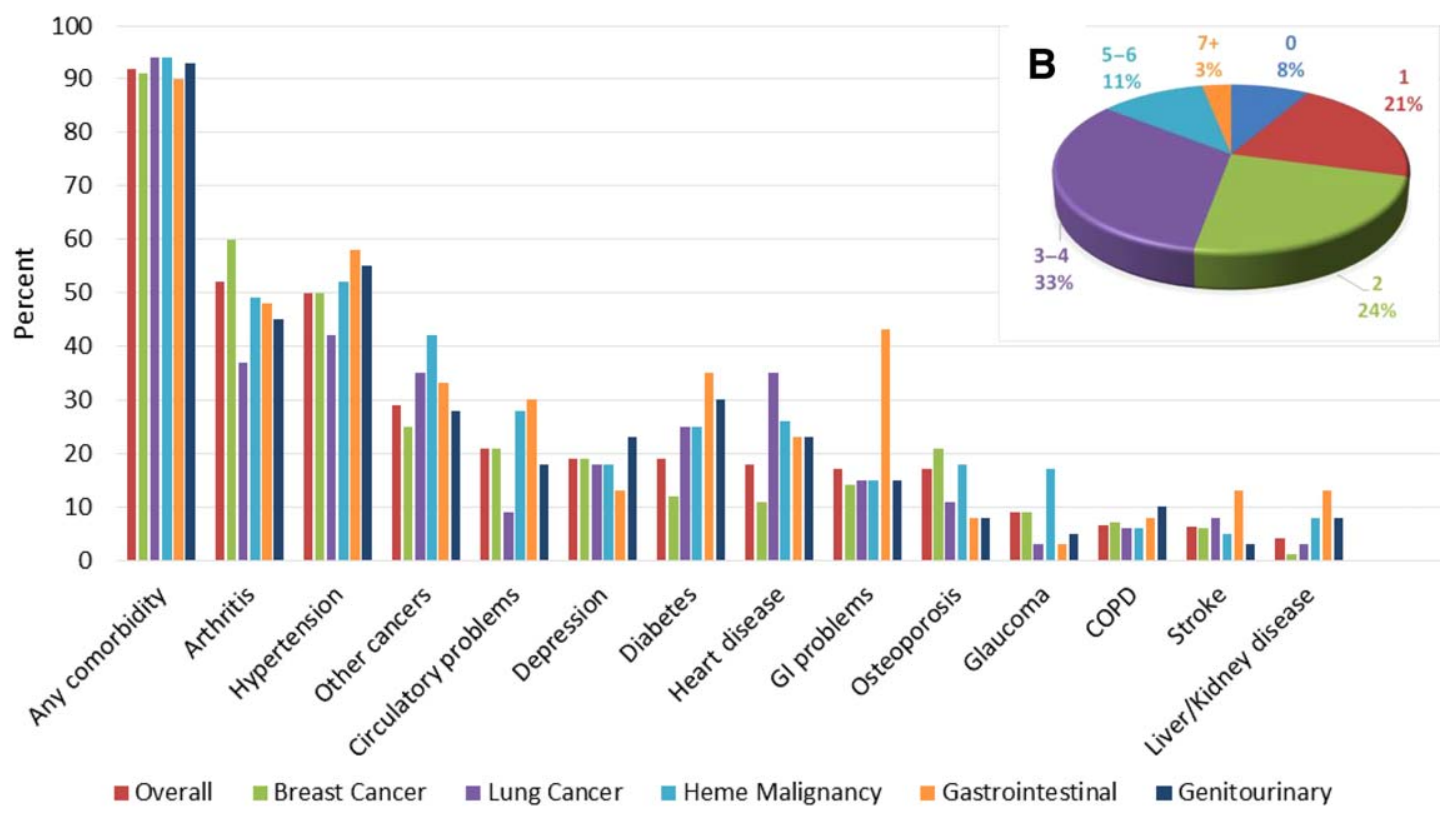

Figure 1. The prevalence of specific comorbid conditions overall and by cancer type (A) and the overall distribution of comorbid conditions (B). Abbreviations: COPD, chronic obstructive pulmonary disease; GI, gastrointestinal.

Comorbidity was defined in three ways: (a) total number of comorbid conditions (ranging from 0 to 13), (b) total number of comorbid conditions interfering with activities (either rated as "somewhat" or "a great deal"; ranging from 0 to 13), and (c) a comorbidity burden score that applied additional weighting for conditions impairing activity. The comorbidity burden score was calculated by multiplying the presence of a comorbid condition by the degree of reported interference with activities ("not at all" =1, "somewhat" = 2, and "a great deal" = 3). The total score for the comorbidity burden score ranged from 0 to 39 .

\section{Statistical Analysis}

We first described the prevalence and distribution of comorbid conditions. The primary outcome of interest was all-cause mortality. Overall survival was measured from the date of completion of the GA to the date of death or last contact as reported by the cancer registry. Cox proportional hazards regression models were used to evaluate the association between comorbidity and all-cause mortality in both the unadjusted and adjusted (for time from diagnosis to GA, age, cancer type, and cancer stage) setting. To examine the influence of comorbidity by cancer stage, we stratified participants by early stage (0-II) and more advanced stages (III/IV) as well. As an exploratory analysis, various models were run to identify potential cutoffs for increased mortality, using a significance level of $p<.05$. Using the lowest identified threshold, overall survival was estimated using the Kaplan-Meier method and unadjusted survival curves were compared using the log-rank test (for the entire cohort and then those participants with the GA performed within 3 months of diagnosis [incident cancer group]). As GAs were completed at different time points throughout the cancer continuum, we performed a subgroup analysis for participants with GA performed within 3 months of diagnosis (incident cancer group). SAS statistical software version 9.4 (SAS Institute Inc., Cary, NC) was used for all analyses.

\section{RESULTS}

\section{Patient Characteristics}

From 2009 to 2014, 703 patients were enrolled into the CSR from the North Carolina Cancer Hospital. Of these patients, 636 were matched to NCCCR using name, date of birth, and sex (90\% match rate), of whom 539 patients were successfully linked to mortality data. The median age at the time of GA was 72 (range 65-100; Table 1). Most patients (85\%) were white and female (72\%). The most common cancer type was breast cancer $(47 \%)$, followed by lung cancer $(12 \%)$, hematologic $(12 \%)$, gastrointestinal $(7 \%)$, and genitourinary malignancies (7\%). The majority had early stage disease $(60 \%$ with stage 0-II). The GA was performed across the cancer continuum, with $33 \%$ having the GA performed within 90 days of diagnosis, 55\% within 1 year, and $45 \%$ with GA performed greater than 1 year from initial diagnosis.

\section{Comorbidity Results}

Ninety-two percent of participants reported at least one comorbid condition. The prevalence of any comorbidity was high across cancer types and the presence of individual comorbid conditions was similar across cancer types (Fig. 1). The median number of comorbid conditions was 2 (range 0-10). Arthritis and hypertension were the most commonly reported comorbidities ( $52 \%$ and $50 \%$, respectively). Sixty-two percent reported an activity limitation related to their comorbidity. Of the reported comorbid conditions impairing activities, chronic obstructive pulmonary disease and arthritis resulted in the most limitations ( $71 \%$ and $69 \%$, respectively). The median comorbidity burden score was 3 (range 0-17).

\section{Comorbidity and All-Cause Mortality}

In the unadjusted setting, when examining comorbidity as a continuous variable, increasing comorbidity was associated 
Table 2. Hazard ratios $(95 \% \mathrm{Cl})$ for all-cause mortality based on patient-reported comorbidity measures as continuous variables ( $n=539$ for all cases, $n=176$ for incident cases)

\begin{tabular}{|c|c|c|c|c|}
\hline Comorbidity measurement & $\begin{array}{l}\text { Unadjusted } \\
\text { hazard ratio } \\
(95 \% \mathrm{Cl})\end{array}$ & $p$ value & $\begin{array}{l}\text { Adjusted }^{\mathrm{a}} \\
\text { hazard ratio }(95 \% \mathrm{Cl})\end{array}$ & $p$ value \\
\hline \multicolumn{5}{|l|}{ All cases } \\
\hline No. of comorbidities & 1.10 (1.02-1.19) & .01 & 1.07 (0.99-1.15) & .09 \\
\hline $\begin{array}{l}\text { No. of comorbidities interfering } \\
\text { with function }\end{array}$ & $1.16(1.06-1.26)$ & $<.001$ & $1.12(1.02-1.23)$ & .02 \\
\hline Comorbidity burden score & $1.07(1.03-1.12)$ & $<.001$ & $1.05(1.01-1.10)$ & .02 \\
\hline \multicolumn{5}{|l|}{ Incident cases } \\
\hline No. of comorbidities & $1.11(0.98-1.26)$ & .11 & 1.17 (0.99-1.39) & .06 \\
\hline $\begin{array}{l}\text { No. of comorbidities interfering } \\
\text { with function }\end{array}$ & $1.14(0.98-1.34)$ & .10 & $1.20(0.97-1.49)$ & .09 \\
\hline Comorbidity burden score & $1.07(1.00-1.14)$ & .06 & $1.12(1.02-1.22)$ & .02 \\
\hline
\end{tabular}

Bolded values are statistically significant $(p<.05)$

${ }^{a}$ Adjusted for time from diagnosis to geriatric assessment, age, cancer type, and stage.

Abbreviation: $\mathrm{Cl}$, confidence interval.

Table 3. Hazard ratios $(95 \% \mathrm{Cl})$ for all-cause mortality based on various thresholds of patient-reported comorbidity using Cox proportional hazards models

\begin{tabular}{lllll}
\hline & $\begin{array}{l}\text { Unadjusted } \\
\text { hazard ratio } \\
(95 \% \text { Cl) }\end{array}$ & $\begin{array}{l}\text { Unadjusted } \\
\boldsymbol{p} \text { value }\end{array}$ & $\begin{array}{l}\text { Adjusted }{ }^{\mathrm{a}} \\
\text { hazard ratio } \\
\text { (95\% Cl) }\end{array}$ & $\begin{array}{l}\text { Adjusted } \\
\boldsymbol{p} \text { value }\end{array}$ \\
\hline $\begin{array}{l}\text { No. of comorbidities } \\
\geq 1 \text { vs. } 0\end{array}$ & $1.34(0.76-2.35)$ & .32 & $1.40(0.76-2.59)$ & .28 \\
$\geq 2$ vs. $\leq 1$ & $1.08(0.78-1.48)$ & .65 & $0.96(0.68-1.36)$ & .84 \\
$\geq 3$ vs. $\leq 2$ & $\mathbf{1 . 4 4 ( 1 . 0 8 - 1 . 9 2 )}$ & .013 & $\mathbf{1 . 4 5 ( 1 . 0 7 - 1 . 9 7 )}$ & .016
\end{tabular}

No. of comorbidities interfering with activities

$\geq 1$ vs. 0

$1.40(1.03-1.90)$

$1.34(0.76-2.35)$

$1.30(0.89-1.88)$

$1.41(1.04-1.93)$

$1.33(1.00-1.78)$

$1.47(1.11-1.96)$
.03

.31

.17

.03

.05

.008
$1.48(1.07-2.05)$

$1.40(0.76-2.59) \quad .28$

$1.17(0.78-1.73) \quad .45$

$1.30(0.93-1.81) \quad .12$

$1.36(1.00-1.84) \quad .05$

1.51(1.13-2.04) $\quad .008$

Bolded values are statistically significant $(p<.05)$

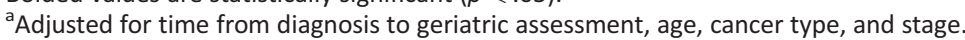

Abbreviation: $\mathrm{Cl}$, confidence interval.

with significantly increased risk of death for all versions of comorbidity measurement (Table 2). There was a stronger association of comorbidity with increased death in participants with earlier stages of cancer (0-II; hazard ratio [HR] 1.24 vs. 1.10 for number of comorbidities, HR 1.28 vs. 1.16 for number interfering with function, and HR 1.14 vs. 1.07 for comorbidity burden score) that was diminished for more advanced stages (III-IV) (HR 1.1 vs. 1.1 for number of comorbidities, HR 1.1 vs. 1.16 for number interfering with function, and HR 1.05 vs. 1.07 for comorbidity burden score). After adjusting for time from diagnosis to GA, age, cancer type, and cancer stage, the risk of death associated with the number of comorbid conditions alone was slightly attenuated (adjusted HR 1.07, confidence interval $[\mathrm{Cl}]$ : 0.99-1.15, $p=.09$ ). Risk of death remained significantly increased for each comorbid condition impacting activities by $12 \%$ (adjusted $\mathrm{HR}=1.12,95 \% \mathrm{Cl}: 1.02-1.23, p=.02$ ) and for each unit increase in comorbidity burden score by $5 \%$ (adjusted HR $=1.05,95 \% \mathrm{Cl}: 1.01-1.10, p=.02$ ). When limiting to just the incident cancer group, only comorbidity burden score remained significantly associated with increased risk of death after controlling for covariates.

In exploratory analyses, we attempted to identify a threshold for increased risk of death for each comorbidity scoring algorithm, and ran multiple models looking at potential thresholds for each (Table 3). For the simple number of comorbidities, patients with three or more total comorbidities had significantly increased risk compared with those with two or fewer (adjusted $\mathrm{HR}=1.45,95 \% \mathrm{Cl}: 1.07-1.97, p=.016)$. For the number of comorbidities interfering with activities, the threshold for increased risk was found at one or more comorbidities (adjusted $\mathrm{HR}=1.48,95 \% \mathrm{Cl}: 1.07-2.05, p=.02$ ), and for comorbidity burden score, a score of 5 or more (adjusted $\mathrm{HR}=1.51,95 \% \mathrm{Cl}: 1.13-2.04, p=.008)$. Figure 2 shows the Kaplan-Meier survival curves for all-cause mortality based on 

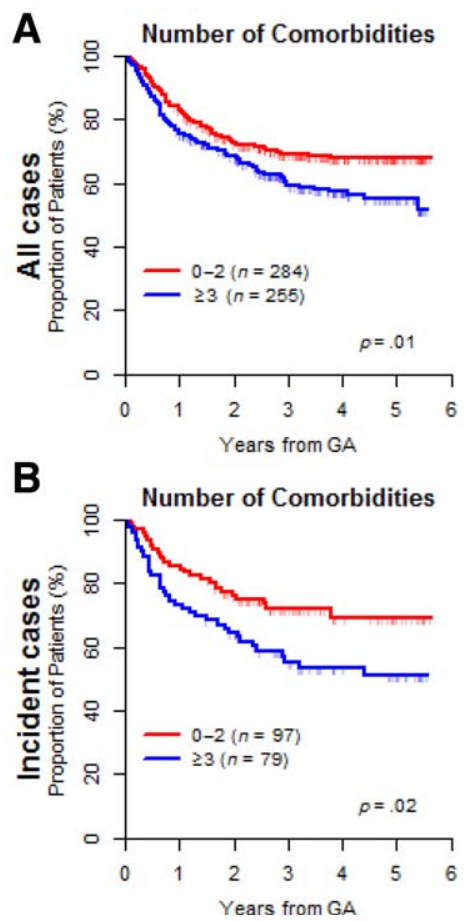
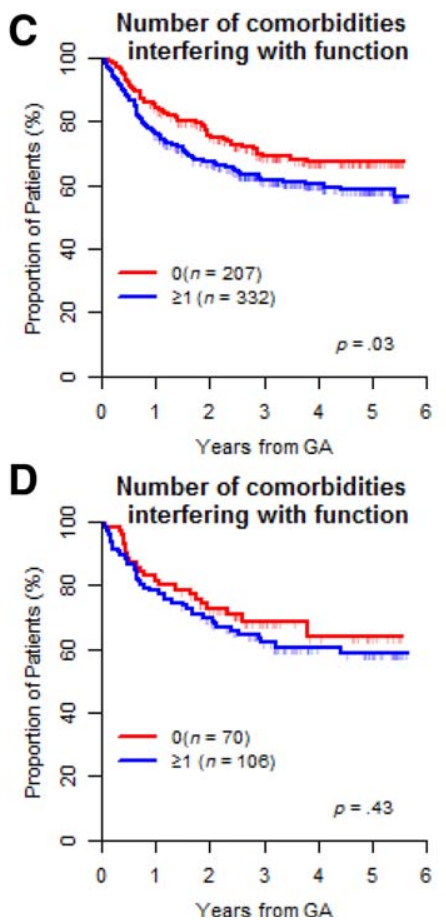
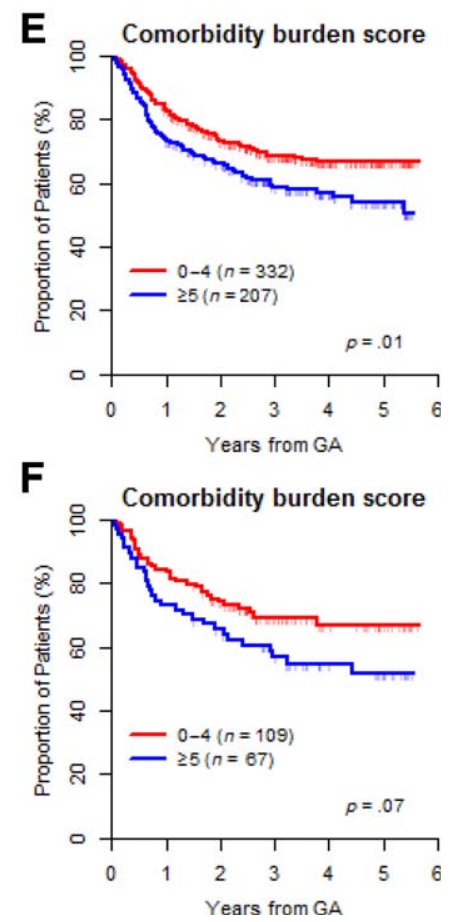

Figure 2. Kaplan-Meier survival curves for all-cause mortality based on patient-reported comorbidity. (A, B): Examine comorbidity based on the overall presence of comorbidity. (C, D): Examine only comorbidities that impact activities. (E, F): Use the comorbidity burden score for all cases (A, C, E) and then for the incident cases (B, D, F). $n=579$ for all cases, and $n=176$ for incident cases.

Abbreviation: GA, geriatric assessment.

these identified thresholds for the number of comorbid conditions, the number of comorbid conditions interfering with activities, and the comorbidity burden score separately for the entire cohort and the incident cancer group.

\section{Discussion}

In our population of older adults with cancer, we confirmed a high prevalence of comorbid conditions (>90\%), with more than half of patients reporting a comorbidity that interferes with activities. Using a patient-reported comorbidity scale, we identified a population of older adults with cancer at increased risk of all-cause mortality. In our adjusted model, the number of comorbid conditions interfering with activities was more strongly associated with an increase in mortality than the presence of comorbid conditions alone. In an exploratory analysis, the presence of three or more comorbid conditions, two or more comorbid conditions interfering with activities, and a comorbidity burden score of 5 or more appeared to be thresholds for increased mortality.

Numerous methods are available for measuring comorbidity in cancer populations [12]; however, systematic comorbidity assessment remains underutilized in cancer clinical trials and clinical practice [6]. The OARS comorbidity measure is a valuable option to assess comorbidity in older adults with cancer. Compared with the commonly used Charlson Comorbidity Index, the OARS results in a broader range of distribution of comorbidity and is able to evaluate the severity of comorbid conditions by assessing the degree to which comorbid conditions interfere with activities [13]. Although less comprehensive than the Cumulative Illness Rating Scale - Geriatric (CIRS-G) [14], its patient-reported format and ease of use makes it particularly suitable to incorporate into clinical trials and practice. However, the OARS comorbidity assessment is limited by only assessing 13 common comorbid conditions. For studies that desire a more comprehensive approach to evaluating comorbidity, the CIRS-G provides a more extensive assessment of comorbid conditions categories by organ system affected with detailed severity scoring $[13,14]$.

Our results are similar to a large number of studies demonstrating poorer survival among patients with cancer and comorbid conditions $[15,16]$. In a review of the impact of comorbidity on cancer survival, Søgaard et al. explored the potential underlying causes of this association. The presence of comorbid conditions is associated with lower rates of surgical management, and multimorbid patients are less likely to receive adjuvant chemotherapy and more likely to receive a reduced dose as well as to not complete chemotherapy when initiated [17]. Whether this represents thoughtful and appropriate treatment modifications by oncology providers based on limited life expectancy remains unclear. Incorporation of comorbidity assessment into clinical trials may help clarify the causes of increased mortality among multimorbid patients. Furthermore, increased comorbidity in all older adults is associated with an increase in all-cause mortality, and older patients with cancer and multiple comorbid conditions are at risk for competing causes of mortality. Competing causes of mortality are an important consideration in older adults with comorbidity when developing treatment plans, particularly in patients with early stage malignancies. Although the association of comorbidity and increased mortality is well established, its relationship with chemotherapy toxicity is less so. In an analysis by Klepin et al. of older women undergoing adjuvant chemotherapy for early stage breast cancer, comorbidity was associated with shorter overall survival, but not chemotherapy toxicity [18]. The two most commonly employed chemotherapy toxicity predictors for use in older adults with cancer do not include 
comorbidity as a variable as there was no significant association of comorbid conditions with severe grade 3/4 chemotherapy toxicity after controlling for functional status, suggesting the impact of comorbidity may be via their effect on function [19, 20]. Comorbid conditions have also been associated with reduced quality of life and impaired functional status in studies examining associations in a cross-section of older adults with cancer $[21,22]$, but less is known about how comorbidity impacts long-term function and quality of life outcomes in those undergoing cancer treatment.

This study has several limitations. First, GAs were completed at different time points throughout the cancer continuum. To take this into account, we included the time from diagnosis to GA completion into our multivariable model as a covariate and performed subgroup analysis separately for the entire cohort and for those with GA performed within 3 months of diagnosis (incident cancer group) for our survival curves. Second, our cohort consists of a heterogeneous cancer population with patients of various cancer types and stages. We examined the prevalence of comorbid conditions separately for each of the more common cancer types and found similar results between cancer types. Although the impact of comorbid conditions on all-cause mortality may vary depending on the specific cancer type of interest, given the small numbers within cancer types, we were unable to examine mortality by individual cancer types. Further work is necessary to refine the prognostic impact of patient-reported comorbidity within specific cancer types and to retest our model to provide further evidence of our thresholds. Third, treatment information is not available in this sample, thus limiting our ability to further examine the impact of comorbidity on type, receipt, and completion of treatment. Fourth, although we were able to successfully link the vast majority of participants to mortality data, approximately 100 participants were lost, and this could result in some selection bias. Lastly, comorbid conditions were selfreported without additional objective testing; however, several studies have shown advantages to patient self-report with good reliability when compared with medical record review $[23,24]$.

\section{CONCLUSION}

Due to changing demographics in the U.S. and worldwide, comorbid conditions are increasingly common in oncology care. It is critical to personalized medicine to understand how coexisting diseases affect cancer outcomes. The optimal care of multimorbid patients with cancer remains unknown and is tremendously limited due to the lack of consistent comorbidity assessment in cancer clinical trials. Developing easy to use and standardized approaches to assessing comorbid conditions is vital to improving the knowledge base to guide the management of these complex patients. Using a simple, patientreported comorbidity questionnaire, the OARS comorbidity scale, we found a high prevalence of comorbidity and developed potential thresholds of increased mortality risk. Comorbid conditions were again demonstrated to affect long-term survival in our population of older adults with cancer; however, the impact of comorbidity on treatment tolerance, functional decline, and quality of life outcomes in older adults with cancer remains less understood. Many unanswered questions remain in optimizing the care of the multimorbid patient with cancer, and the first step toward improving care for these vulnerable patients is through routine comorbidity assessments. Embracing patient-reported comorbidity assessment may improve the incorporation of comorbidity assessment without additional resource allocation.

\section{AcKNOWLEDGMENTS}

Previously presented as a poster at the American Society of Clinical Oncology annual meeting, Chicago, IL, June 3, 2017.

This work was supported in part by the University Cancer Research Fund at The University of North Carolina and the Clinical and Translational Science Award program of the National Center for Advancing Translational Sciences, National Institutes of Health (1UL1TR001111).

\begin{abstract}
Author Contributions
Conception/design: Grant R. Williams, Allison M. Deal, Jennifer L. Lund, Emily J. Guerard, Hanna K. Sanoff

Data analysis and interpretation: Grant R. Williams, Allison M. Deal, Jennifer L. Lund, YunKyung Chang, Hyman B. Muss, Mackenzi Pergolotti, Emily J. Guerard, Shlomit Strulov Shachar, Yue Wang, Kelly Kenzik, Hanna K. Sanoff

Manuscript writing: Grant R. Williams

Final approval of manuscript: Grant R. Williams, Allison M. Deal, Jennifer L. Lund, YunKyung Chang, Hyman B. Muss, Mackenzi Pergolotti, Emily J. Guerard, Shlomit Strulov Shachar, Yue Wang, Kelly Kenzik, Hanna K. Sanoff
\end{abstract}

\section{DiscLOSURES}

Jennifer L. Lund: GlaxoSmithKline (E [spouse]). The other authors indicated no financial relationships.

(C/A) Consulting/advisory relationship; (RF) Research funding; (E) Employment; (ET) Expert testimony; (H) Honoraria received; (OI) Ownership interests; (IP) Intellectual property rights/ inventor/patent holder; (SAB) Scientific advisory board

\section{REFERENCES}

1. Smith BD, Smith GL, Hurria A et al. Future of cancer incidence in the United States: Burdens upon an aging, changing nation. J Clin Oncol 2009;27:27582765.

2. Ritchie CS, Kvale E, Fisch MJ. Multimorbidity: An issue of growing importance for oncologists. J Oncol Pract 2011;7:371-374.

3. Wolff JL, Starfield B, Anderson G. Prevalence, expenditures, and complications of multiple chronic conditions in the elderly. Arch Intern Med 2002;162: 2269-2276.

4. Jorgensen TL, Hallas J, Friis S et al. Comorbidity in elderly cancer patients in relation to overall and cancer-specific mortality. Br J Cancer 2012;106: 1353-1360.
5. Koroukian SM, Murray P, Madigan E. Comorbidity, disability, and geriatric syndromes in elderly cancer patients receiving home health care. J Clin Oncol 2006;24:2304-2310.

6. Williams GR, Mackenzie A, Magnuson A et al Comorbidity in older adults with cancer. J Geriatr Oncol 2016;7:249-257.

7. Basch E, Snyder C, McNiff $K$ et al. Patientreported outcome performance measures in oncology. J Oncol Pract 2014;10:209-211.

8. Fillenbaum GG, Smyer MA. The development, validity, and reliability of the OARS multidimensional functional assessment questionnaire. J Gerontol 1981;36:428-434.
9. Williams GR, Deal AM, Jolly TA et al. Feasibility of geriatric assessment in community oncology clinics. J Geriatr Oncol 2014;5:245-251.

10. Hurria A, Gupta S, Zauderer M et al. Developing a cancer-specific geriatric assessment: a feasibility study. Cancer 2005;104:1998-2005.

11. Lund JL, Meyer AM, Deal AM et al. Data linkage to improve geriatric oncology research: A feasibility study. The Oncologist 2017;22:1002-1005.

12. Sarfati D. Review of methods used to measure comorbidity in cancer populations: No gold standard exists. J Clin Epidemiol 2012;65:924-933.

13. Extermann M. Measuring comorbidity in older cancer patients. Eur J Cancer 2000;36:453-471. 
14. Miller MD, Paradis CF, Houck PR et al. Rating chronic medical illness burden in geropsychiatric practice and research: Application of the Cumulative Illness Rating Scale. Psychiatry Res 1992;41:237248.

15. Sogaard M, Thomsen RW, Bossen KS et al. The impact of comorbidity on cancer survival: A review. Clin Epidemiol 2013;5(suppl 1):3-29.

16. Extermann M. Measurement and impact of comorbidity in older cancer patients. Crit Rev Oncol Hematol 2000;35:181-200.

17. Smith SM, Soubhi H, Fortin $M$ et al. Interventions for improving outcomes in patients with multimorbidity in primary care and community settings. Cochrane Database Syst Rev 2012; 18:CD006560.
18. Klepin HD, Pitcher BN, Ballman KV et al. Comorbidity, chemotherapy toxicity, and outcomes among older women receiving adjuvant chemotherapy for breast cancer on a clinical trial: CALGB 49907 and CALGB 361004 (Alliance). J Oncol Pract 2014;10: e285-e292.

19. Extermann M, Boler I, Reich RR et al. Predicting the risk of chemotherapy toxicity in older patients: The Chemotherapy Risk Assessment Scale for High-Age Patients (CRASH) score. Cancer 2012;118:3377-3386.

20. Hurria A, Togawa K, Mohile SG et al. Predicting chemotherapy toxicity in older adults with cancer: A prospective multicenter study. J Clin Oncol 2011;29: 3457-3465.

21. Pergolotti M, Deal AM, Williams GR et al. Activities, function, and health-related quality of life
(HRQOL) of older adults with cancer. J Geriatr Oncol 2017;8:249-254.

22. Garman KS, Pieper CF, Seo $P$ et al. Function in elderly cancer survivors depends on comorbidities. J Gerontol A Biol Sci Med Sci 2003;58:M1119-M1124.

23. Kriegsman DM, Penninx BW, van Eijk JT et al. Self-reports and general practitioner information on the presence of chronic diseases in community dwelling elderly. A study on the accuracy of patients' self-reports and on determinants of inaccuracy. J Clin Epidemiol 1996;49:1407-1417.

24. Ye F, Moon DH, Carpenter WR et al. Comparison of patient report and medical records of comorbidities: Results from a population-based cohort of patients with prostate cancer. JAMA Oncol 2017;3: 1035-1042.

\section{For Further Reading:}

Trevor A. Jolly, Allison M. Deal, Kirsten A. Nyrop et al. Geriatric Assessment-Identified Deficits in Older Cancer Patients With Normal Performance Status. The Oncologist 2015;20:379-385; first published on March 12, 2015.

\section{Implications for Practice:}

The optimal evaluation to guide treatment decisions for older cancer patients is not known. The Karnofsky performance status (KPS) scale is frequently used to guide oncology practice, whereas the standard in geriatric medicine is the comprehensive geriatric assessment (GA). Comprehensive GA is time and resource intensive and impractical in routine cancer care. This study shows that a brief, mostly patient-administered GA can identify deficits that could affect treatment tolerance and outcomes in patients assessed as functionally normal by KPS. A brief GA should be incorporated into routine oncology practice for timely identification of patient deficits that may be remediable before or during treatment. 